\title{
Accurate monitoring of promoter gene methylation with high-resolution melting polymerase chain reaction using the $A B C B 1$ gene as a model
}

\author{
A.L. Mencalha ${ }^{1,2}$, E.F. Rodrigues ${ }^{1}$, E. Abdelhay ${ }^{1}$ and T.S. Fernandez ${ }^{1}$ \\ ${ }^{1}$ Centro de Estudos de Medula Óssea, Instituto Nacional de Câncer, \\ Rio de Janeiro, RJ, Brasil \\ ${ }^{2}$ Departamento de Biofísica e Biometria, \\ Instituto de Biologia Roberto Alcântara Gomes, \\ Universidade do Estado do Rio de Janeiro, Rio de Janeiro, RJ, Brasil \\ Corresponding authors: A.L. Mencalha / T.S. Fernandez \\ E-mail: amencalha@inca.gov.br / teresafernandez@inca.gov.br
}

Genet. Mol. Res. 12 (1): 714-722 (2013)

Received August 21, 2012

Accepted December 21, 2012

Published March 11, 2013

DOI http://dx.doi.org/10.4238/2013.March.11.20

\begin{abstract}
Multidrug resistance is the major cause of cancer chemotherapy failure. This phenotype is mainly due to the overexpression of the human $A B C B 1$ gene. Several studies have shown that the transcriptional regulation of this gene is complex. Yet, the impact of this transcriptional regulation has not been well studied in a clinical setting. The acquired expression of $A B C B 1$ is associated with the genomic instability of cancer cells. This includes the occurrence of mutational events that alter chromatin structures through epigenetic modifications such as promoter methylation. Therefore, it is important to introduce new clinical methods to monitor the methylation status of $A B C B 1$ and determine its association with gene expression in order to be able to predict response to therapies. The high-resolution melting (HRM) method has emerged as a highly accurate and sensitive
\end{abstract}


method to quantify methylation status at specific sites of DNA. Here, we established HRM parameters to evaluate the promoter methylation status of the $A B C B 1$ gene. Our study is the first to standardize the HRM dissociation curve to evaluate $A B C B 1$ gene methylation. The association between $A B C B 1$ methylation status and gene expression in established cancer cell lines shows that this method is accurate and reliable.

Key words: $A B C B 1$; HRM-PCR; Methylation; Epigenetics

\section{INTRODUCTION}

ATP-binding cassette $(\mathrm{ABC})$ transporter family genes function through a common mechanism to drive multidrug resistance (MDR). Among them is the $A B C B 1$ gene, also known as $M D R-1$, which encodes a P-glycoprotein (Pgp) that it is universally accepted as a drug resistance biomarker (Ueda et al., 1987; Wise, 2012). Pgp is a trans-membrane protein that is responsible for the active efflux of anticancer drugs from cells (Ueda et al., 1987). In cancer cells, Pgp is frequently overexpressed (Goldie and Coldman, 1984). Primary or acquired overexpression of $A B C B 1$ is the major cause of resistance to chemotherapy and is associated with reduced patient survival (Abolhoda et al., 1999). The overexpression of the $A B C B 1$ gene is negatively correlated with disease prognosis and quality of life (Baker et al., 2005). Therefore, strategies to overcome drug resistance have been actively sought for years. However, currently, no efficient clinical solutions exist to counter drug resistance.

The acquired expression of $A B C B 1$ is associated with the genomic instability of cancer cells. This includes mutational events that alter chromatin structures and cause gene rearrangements and mutations in tumor suppressor proteins like P53 (Kuo et al., 1994). In addition, epigenetic modifications of the $A B C B 1$ proximal and upstream promoters, either through DNA demethylation or through histone $\mathrm{H} 3$ acetylation, play a pivotal role in inducing $A B C B 1$ expression (Chen and Sikic, 2012). Therefore, a better understanding of $A B C B 1$ methylation status can improve the management of therapies in an effort to increase treatment efficacy.

The proximal promoter region directly upstream of the $A B C B 1$ transcription start site contains several important consensus regulatory sequences, including CAAT motifs and $\mathrm{CpG}$ islands, which are implicated in gene expression in response to different stimuli (Ueda et al., 1987; El-Osta et al., 2002). Several lines of evidence suggest that the methylation of the $\mathrm{CpG}$ dinucleotides in the $A B C B 1$ gene plays a major role in regulating its expression. The $A B C B 1$ gene-regulatory region extends from the start of exon 1 to a few base pairs upstream of the transcriptional start site (Baker et al., 2005). The up-regulation of $A B C B 1$ mRNA has been associated with the hypomethylation of the promoter region, whereas the down-regulation of $A B C B 1$ expression is associated with the hypermethylation of the $\mathrm{CpG}$ dinucleotides (Kantharidis et al., 1997; Nakayama et al., 1998; Kusaba et al., 1999). Although promoter methylation of $A B C B 1$ is associated with its expression level, no molecular methods have shown the relationship between the level of promoter methylation of this gene and gene expression.

In 2003, a highly sensitive method to identify a single nucleotide change in a specific DNA sequence was described (Wittwer et al., 2003). This method determines DNA changes based on the fine melting temperature properties of an amplicon of the sequence of interest. This method is called high-resolution melting (HRM). HRM requires specific thermocycler platforms and a 
saturating DNA intercalating dye. This method has been used to quantify methylation in CpG islands (Wojdacz and Dobrovic, 2007). The difference between methylated and unmethylated DNA is determined by bisulfite sodium treatment. Whereas non-methylated cytosines are converted into uracil nucleotides upon bisulfite treatment and are substituted by thymidine nucleotides in polymerase chain reaction (PCR), the 5-methyl-cytosines are not converted, thus are read as cytosine. The cytosines in $\mathrm{CpG}$ dinucleotides cause differences in melting temperature between methylated and unmethylated DNA (Wojdacz and Dobrovic, 2007). Unlike other methods used for the quantification of methylation levels, HRM is faster, less expensive, highly accurate, and sensitive.

In the present study, we established a strategy to quantify DNA methylation using the HRM method. We used the $A B C B 1$ gene as a model because of its importance in the MDR phenotype. We first identified the $\mathrm{CpG}$ islands in the DNA upstream of the $A B C B 1$ gene and determined parameters to accurately quantify methylation status in this region by HRM. We also analyzed the association between the methylation status and the mRNA expression level of the $A B C B 1$ gene in different neoplastic cell lines. We observed an inverse correlation between the level of methylation and gene expression.

\section{MATERIAL AND METHODS}

\section{Cell lines}

The cell lines used in this study were as follows: the K562 cell line, established from a chronic myeloid leukemia patient in blast crisis; the DLD-1 cell line, established from colorectal adenocarcinoma; the Raji cell line, established from Burkitt's lymphoma; the HCC-1954 cell line, established from breast ductal carcinoma, and the MCF-7 cell line, isolated and established from breast cancer. The K562 and Raji cells were cultured in Roswell Park Memorial Institute (RPMI) 1640 medium (Invitrogen, USA). The DLD-1, MCF-7, and HCC-1954 cells were cultured with Dulbecco's modified Eagle's medium (Invitrogen). All cultures were supplemented with $10 \%$ fetal bovine serum (Hyclone, USA), $100 \mathrm{U} / \mathrm{mL}$ penicillin (Invitrogen), and $100 \mathrm{mg} / \mathrm{mL}$ streptomycin (Invitrogen) and cultured at $37^{\circ} \mathrm{C}$ in $5 \% \mathrm{CO}_{2}$.

\section{DNA sequence analysis}

The $A B C B 1$ gene sequence was obtained from the National Center for Biotechnology Information (accession No. NG_011513). CpG islands were predicted using the online tool MethPrimer using default parameters (Li and Dahiya, 2002).

\section{DNA extraction and bisulfite treatment}

The cell lines were washed 3 times in $1 \mathrm{X}$ phosphate-buffered saline and subsequently the cell lysates were used for genomic DNA extraction using DNAzol (Invitrogen) according to the manufacturer protocol. A total of $2 \mu \mathrm{g}$ genomic DNA was used for bisulfite modification using the Epitect Bisulfite kit (Qiagen, USA) according to manufacturer instructions. Reactions were performed in a $140-\mu \mathrm{L}$ volume with $85 \mu \mathrm{L}$ bisulfite mix and $35 \mu \mathrm{L}$ DNA protection buffer. The thermal cycler conditions were: $5 \mathrm{~min}$ at $95^{\circ} \mathrm{C}, 25 \mathrm{~min}$ at $60^{\circ} \mathrm{C}, 5 \mathrm{~min}$ at $95^{\circ} \mathrm{C}, 85$ min at $60^{\circ} \mathrm{C}, 5 \mathrm{~min}$ at $95^{\circ} \mathrm{C}, 75 \mathrm{~min}$ at $60^{\circ} \mathrm{C}$, and a final hold at $20^{\circ} \mathrm{C}$. After bisulfite conversion 
was completed, the DNA was cleaned and eluted once in $20 \mu \mathrm{L}$ elution buffer. The DNA was either used immediately or stored at $-70^{\circ} \mathrm{C}$ to avoid degradation.

\section{High-resolution melting}

PCR cycling was performed on the Rotor-Gene Q (Qiagen) HRM-enabled real-time PCR instrument. Primers were designed according to the principles of HRM from previous studies (Toyota et al., 2001; Wojdacz et al., 2009). PCR was performed in a final volume of $10 \mu \mathrm{L}$, with 1 $\mu \mathrm{M}$ of each primer, $10 \mathrm{ng}$ bisulfite-converted DNA, and 1X EpiTect HRM Master Mix (Qiagen). The standard curve to quantify methylation percentages were established at 100, 75, 50, 25, 10, and 5\% DNA methylation using the Epitect control DNA set (Qiagen). PCR cycling was carried out as follows: initial denaturation at $95^{\circ} \mathrm{C}$ for $10 \mathrm{~min}$, followed by 45 cycles of $45 \mathrm{~s}$ at $95^{\circ} \mathrm{C}, 45$ $\mathrm{s}$ at $60^{\circ} \mathrm{C}$, and $45 \mathrm{~s}$ at $72^{\circ} \mathrm{C}$. After amplification, the PCR product was pre-heated for $90 \mathrm{~s}$ at $60^{\circ} \mathrm{C}$ followed by the HRM step, which involved gradually increasing the incubation temperature from $65^{\circ}$ to $90^{\circ} \mathrm{C}$ at a rate of $0.1^{\circ} \mathrm{C} / \mathrm{s}$ and holding for $2 \mathrm{~s}$ after each stepwise increment. Melting curves from methylated and unmethylated DNA controls were normalized by selecting the "line of best fit" between 2 normalization regions selected before and after obtaining the raw data of the dissociation curve to generate a better profile of methylation percentages (Wojdacz and Dobrovic, 2007). The HRM analyses were performed as previously described (Wojdacz et al., 2009). The $A B C B 1$ DNA promoter region was amplified using primers that include $\mathrm{CpG}$ islands from $-19 \mathrm{bp}$ to $+146 \mathrm{bp}$ from the transcription start site: $A B C B 1$-HRM-forward: 5'-GTTATAGGAAGTTTGAGTTT-3' and $A B C B 1$-HRM-reverse: 5'-AAAAACTATCCCATAATAAC-3'.

\section{Quantitative PCR (qPCR)}

Changes in $A B C B 1$ mRNA levels were measured by qPCR. Total mRNA was obtained from all cell lines using the TRIzol reagent (Invitrogen) according to the manufacturer protocol. The mRNA was stored at $-70^{\circ} \mathrm{C}$. Two micrograms of total RNA was subjected to genomic DNA digestion with DNAse, amplification grade I (Invitrogen) to remove genomic DNA contamination. The RNA was reverse transcribed to complementary DNA (cDNA) with the Superscript II Reverse Transcriptase (Invitrogen) and Oligo-dT18 (Invitrogen) kits. Reactions were performed in $10 \mu \mathrm{L}$ with a final concentration of 1X Rotor-Gene SYBR Green PCR Kit (Qiagen). Forward and reverse primers were used at a concentration of $0.5 \mu \mathrm{M}$ each and were mixed with $2.5 \mu \mathrm{L}$ 5-fold diluted cDNA. Reactions were carried out in a Rotor Gene Q thermocycler (Qiagen) with a hot-start stage step of $10 \mathrm{~min}$ at $95^{\circ} \mathrm{C}$ followed by 45 cycles of $20 \mathrm{~s}$ at $95^{\circ} \mathrm{C}$, $30 \mathrm{~s}$ at $60^{\circ} \mathrm{C}$, and $30 \mathrm{~s}$ at $72^{\circ} \mathrm{C}$. The dissociation curve was used to determine PCR efficiency, specificity of amplification, and primer-dimer formation. $\beta$-actin mRNA levels were used as a reference for normalization. Sequences of the primers used are as follows: $A B C B 1$ forward: 5'-TCGTGCCCTTGTTAGACAG-3', ABCB1 reverse: 5'-CATTCTGGATGGTGGACAGG3', $\beta$-actin forward: 5'-TTCCTTCCTGGGCATGGAGTC-3', and $\beta$-actin reverse: 5'-AGACA GCACTGTGTTGGCGTA-3'.

\section{Statistical analyses}

The significance of the DNA methylation percentage was determined by comparing 
the differences between curve shapes for methylated and unmethylated DNA and it was estimated by principal component analysis using a set of DNA controls as the reference. Correlation analysis between methylation and mRNA levels was performed using the Pearson test with GraphPad Prism version 5 (GraphPad Software, Inc., USA).

\section{RESULTS}

\section{Identification of $\mathrm{CpG}$ islands in the $A B C B 1$ gene and validation of the HRM method to analyze methylation status using the $A B C B 1$ gene as a model}

We investigated the promoter region of the $A B C B 1$ gene to identify $\mathrm{CpG}$ islands using the MethPrimer online software. We focused on approximately $2000 \mathrm{bp}$, in which -1794 bp are from the transcription start site of $A B C B 1$ up to the first $+205 \mathrm{bp}$ in exon 1. In this region, we found a $\mathrm{CpG}$ island near the transcription start site in exon 1 . According to the MethPrimer software, this island extends from nucleotides -182 to +146 . Approximately $21 \mathrm{CpGs}$ were identified in this predicted island. This result is shown in Figure 1.

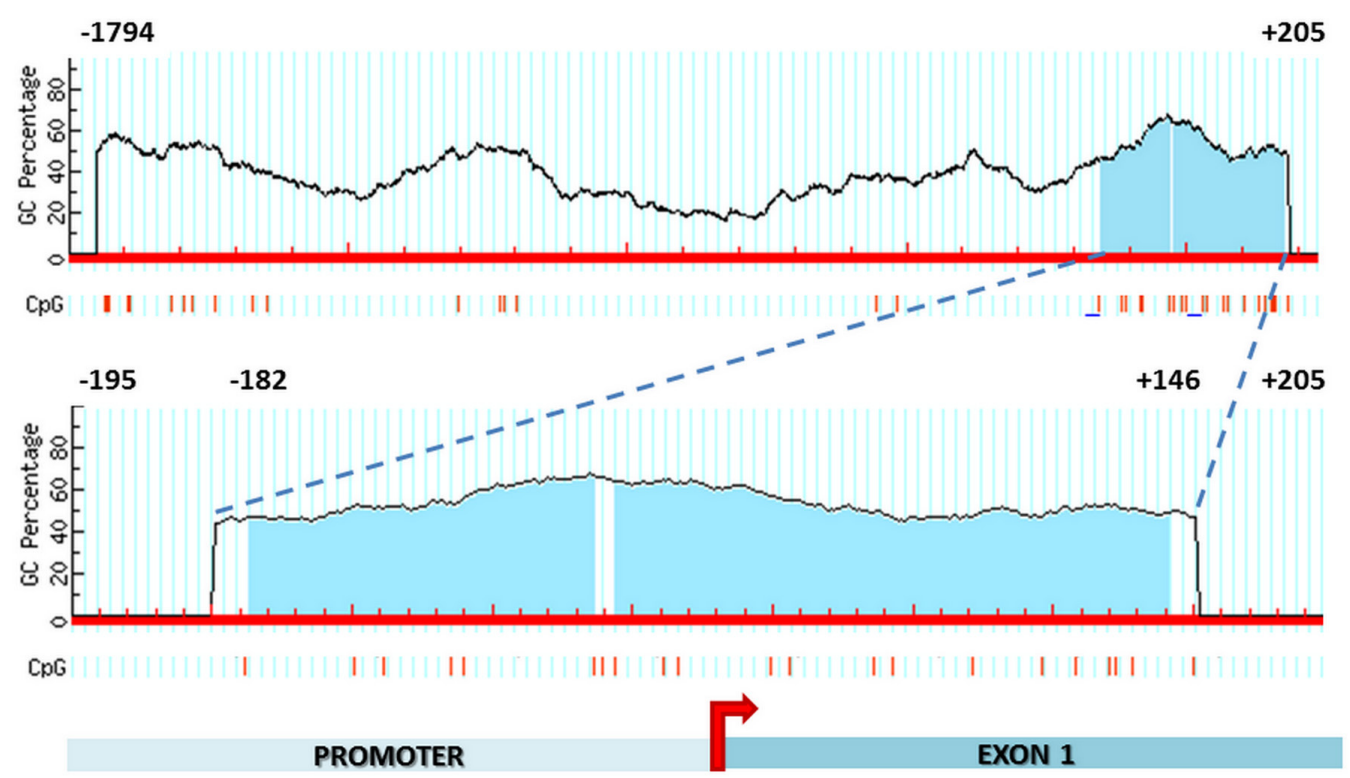

Figure 1. Determination of CpG island at 2000 bp (-1794 up to +205 bp) (upper panel) and 400 bp ( -195 up to +205 bp) (lower panel) of DNA sequence near the $A B C B 1$ gene. Filled light blue areas indicate the predicted $\mathrm{CpG}$ island. The red fine bars under $\mathrm{x}$-axis panels indicate the isolate $\mathrm{CpG}$ dinucleotide along the promoter $A B C B 1$ DNA region. Figure was adapted from the MethPrimer Software (Li and Dahiya, 2002).

To evaluate the association between the methylation status of the $A B C B 1$ gene and its mRNA level, we performed a paired analysis between the methylation percentage of $A B C B 1$ as determined by HMR and $A B C B 1$ mRNA expression as determined by qPCR analysis in established cell lines. The normalization and melting curves were generated with 100, 75, 50, $25,10,5$, and $0 \%$ of methylated DNA. These results are shown in Figures 2 and 3. With this 
standard curve, it is possible to estimate the percentage of $A B C B 1$ methylation in any cellular sample derived from human cell lines or directly from patients. Our HRM results showed that the K562 cells had a low percentage of $A B C B 1$ gene methylation, approximately $5 \%$, and that the $A B C B 1$ downstream promoter was unmethylated in DLD-1 cells. On the other hand, the cell lines Raji, HCC-1954, and MCF-7 showed approximately 50, 50-75, and 75-100\% $A B C B 1 \mathrm{CpG}$ island methylation, respectively. These results are shown in Figures 2 and 3.
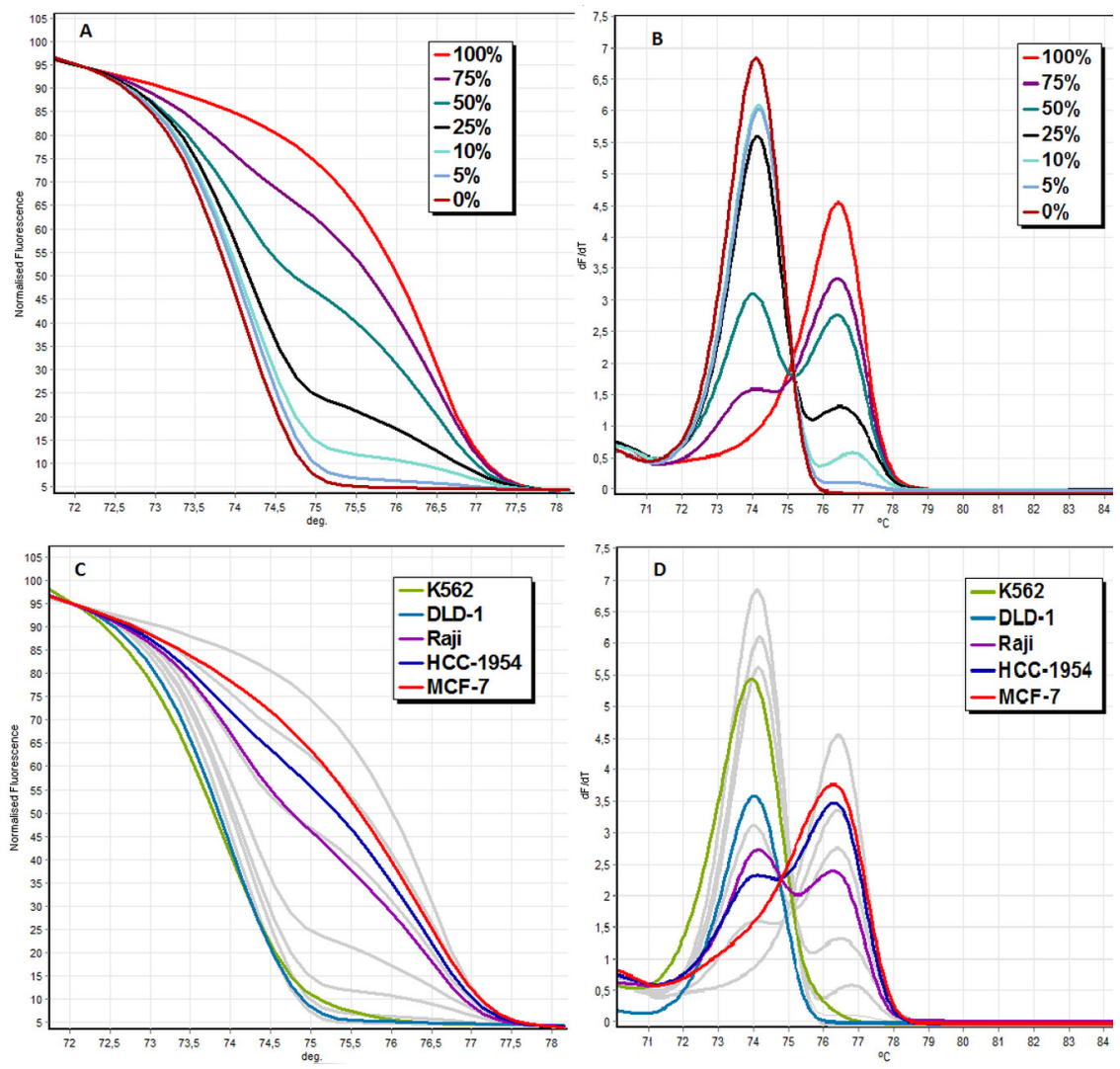

Figure 2.A. High-resolution melting analysis: normalized standard curves from $100,75,50,25,10,5$, and $0 \%$ methylation using DNA standard set. B. Dissociation curves from 100, 75, 50, 25, 10, 5, and 0\% methylation using DNA standard set. C. and D. Methylation estimatives of K562, DLD-1, Raji, HCC-1952, and MCF-7 cell lines are represented by colored curves and the standard DNA. In all panels the normalized and dissociation set curves from methylation DNA controls are represented in gray curves. Data were edited in TeeChart Office 2.0, Steema Software data.

The expression analyses by qPCR of the $A B C B 1$ gene in K562 and DLD-1 cells showed elevated mRNA levels in both cells: 9024 [ \pm standard deviation (SD) 555] and $13,113 \pm 577$ copies, respectively. However, the analysis of $A B C B 1$ mRNA expression in Raji, HCC-1954, and MCF-7 cells showed low levels of $A B C B 1$ mRNA: $842 \pm 154,261 \pm$ 27 , and $42 \pm 7$ copies, respectively. Pearson's correlation analysis, comparing the percentage of DNA methylation with mRNA expression levels in the cell lines, indicated a strong 
inverse correlation $(-0.945, \mathrm{P}=0.015)$ between the two measures. These results are shown in Figure 3.

DNA METHYLATION (\%)

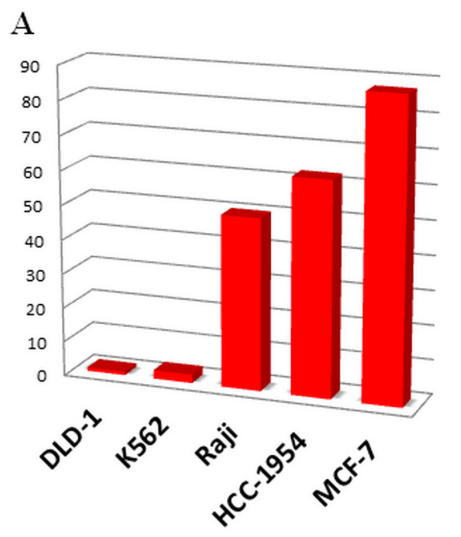

RELATIVE mRNA LEVELS

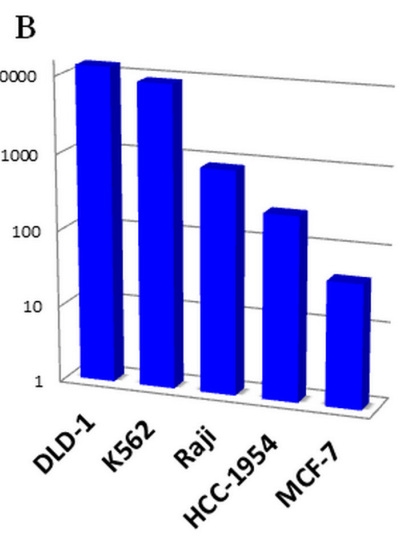

LINEAR CORRELATION (Methylation vs mRNA levels)

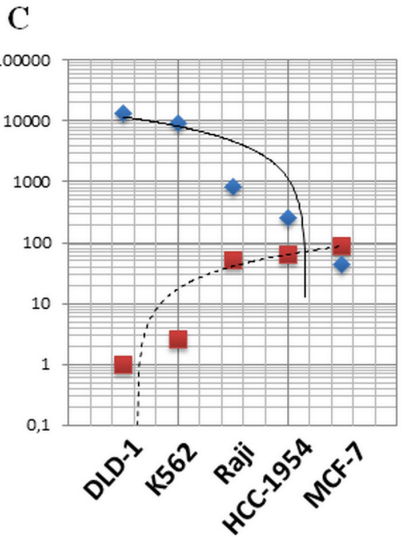

Figure 3. Comparison between the percent of $A B C B 1$ DNA promoter methylation and $A B C B 1$ mRNA relative levels. A. Methylation percentage. B. Relative units of mRNA level. C. Linear correlation between methylation versus mRNA levels. Squares and dotted line represent methylation data, and lozenges and continuous line represent mRNA level data. Data were analyzed and edited in GraphPad Prism version 5 and Excel Microsoft, 2010.

\section{DISCUSSION}

DNA methylation is responsible for controlling about $60 \%$ of transcription (Suzuki and Bird, 2008). This process is regulated by DNA methylation of the $\mathrm{CpG}$ islands that are frequently found near the transcription start site and in the first exon and first intron (Suzuki and Bird, 2008). Several studies have revealed the presence of $\mathrm{CpG}$ islands from the first intron of $A B C B 1$ to a few nucleotides upstream of the transcription start site (Nakayama et al., 1998). These DNA regions have been reported as targets of methylation (Jin and Scotto, 1998; Ando et al., 2000). Although a few studies have addressed the association between $A B C B 1$ methylation status and the expression of this gene, the precise mechanism of $A B C B 1$ gene expression regulation is not yet clear (Kantharidis et al., 1997; Nakayama et al., 1998; Toyota et al., 2001; Backer et al., 2005; Kim et al., 2009).

$A B C B 1$ expression is frequently implicated in several pathologies, especially in cancer, as a hallmark of multidrug resistance. However, HRM has not yet been used to evaluate the percentage of methylation in this gene and the association of methylation percentage with gene expression. A previous study indicated that in $\mathrm{K} 562$ cells the $A B C B 1$ promoter region had lowly methylated or unmethylated DNA and that $A B C B 1$ gene expression was correlated to its methylation status (Ando et al., 2000). Additionally, DLD-1 cells also showed a correlation between the status of $A B C B 1$ gene methylation and gene expression (Kim et al., 2009). In MCF-7 cells, the $A B C B 1$ expression level was inversely correlated with the methylation 
status of the $A B C B 1$ downstream promoter (Reed et al., 2010). Our results showed that the MCF-7 cell line had the highest percentage of DNA methylation among the cell lines that we studied and that it had low $A B C B 1$ expression. A previous study showed that the genome of the HCC-1954 cell line was predominantly hypomethylated with specific DNA regions being hypermethylated (Hon et al., 2012). There are no studies showing a correlation between the methylation status and expression of the $A B C B 1$ gene in the HCC-1954 cell line. However, our results suggest that the promoter region of the $A B C B 1$ gene is part of the region of the HCC1954 genome that is hypermethylated and that this hypermethylation status is associated with low levels of $A B C B 1$ transcripts.

Our study is the first to standardize the HRM dissociation curve to evaluate $A B C B 1$ gene methylation status. The association between $A B C B 1$ methylation status and gene expression in established cancer cell lines shows that this method is accurate and reliable. This method can be used to study the MDR phenotype of different human samples, and can thus assist in the choice of therapeutic protocols used in patients with cancer or other diseases. Therefore, our HRM analysis is useful for determining $A B C B 1$ DNA methylation and for predicting $A B C B 1$ expression.

\section{Conflict of interest}

All authors declare no competing interests.

\section{ACKNOWLEDGMENTS}

Research supported by grants from Fundação de Amparo à Pesquisa do Estado do Rio de Janeiro/FAPERJ (\#E-26/102.235/2009), Conselho Nacional de Desenvolvimento Científico e Tecnológico/CNPq (\#478564/2011-2), and Instituto Nacional de Câncer/INCA.

\section{REFERENCES}

Abolhoda A, Wilson AE, Ross H, Danenberg PV, et al. (1999). Rapid activation of MDR1 gene expression in human metastatic sarcoma after in vivo exposure to doxorubicin. Clin. Cancer Res. 5: 3352-3356.

Ando T, Nishimura M and Oka Y (2000). Decitabine (5-Aza-2'-deoxycytidine) decreased DNA methylation and expression of MDR-1 gene in K562/ADM cells. Leukemia 14: 1915-1920.

Baker EK, Johnstone RW, Zalcberg JR and El-Osta A (2005). Epigenetic changes to the MDR1 locus in response to chemotherapeutic drugs. Oncogene 24: 8061-8075.

Chen KG and Sikic BI (2012). Molecular pathways: regulation and therapeutic implications of multidrug resistance. Clin. Cancer Res. 18: 1863-1869.

El-Osta A, Kantharidis P, Zalcberg JR and Wolffe AP (2002). Precipitous release of methyl-CpG binding protein 2 and histone deacetylase 1 from the methylated human multidrug resistance gene (MDR1) on activation. Mol. Cell Biol. 22: $1844-1857$.

Goldie JH and Coldman AJ (1984). The genetic origin of drug resistance in neoplasms: implications for systemic therapy. Cancer Res. 44: 3643-3653.

Hon GC, Hawkins RD, Caballero OL, Lo C, et al. (2012). Global DNA hypomethylation coupled to repressive chromatin domain formation and gene silencing in breast cancer. Genome Res. 22: 246-258.

Jin S and Scotto KW (1998). Transcriptional regulation of the MDR1 gene by histone acetyltransferase and deacetylase is mediated by NF-Y. Mol. Cell Biol. 18: 4377-4384.

Kantharidis P, El-Osta A, deSilva M, Wall DM, et al. (1997). Altered methylation of the human MDR1 promoter is associated with acquired multidrug resistance. Clin. Cancer Res. 3: 2025-2032.

Kim SN, Kim NH, Lee W, Seo DW, et al. (2009). Histone deacetylase inhibitor induction of P-glycoprotein transcription 
requires both histone deacetylase 1 dissociation and recruitment of CAAT/enhancer binding protein beta and pCAF to the promoter region. Mol. Cancer Res. 7: 735-744.

Kuo MT, Vyas RC, Jiang LX and Hittelman WN (1994). Chromosome breakage at a major fragile site associated with P-glycoprotein gene amplification in multidrug-resistant CHO cells. Mol. Cell Biol. 14: 5202-5211.

Kusaba H, Nakayama M, Harada T, Nomoto M, et al. (1999). Association of 5' CpG demethylation and altered chromatin structure in the promoter region with transcriptional activation of the multidrug resistance 1 gene in human cancer cells. Eur. J. Biochem. 262: 924-932.

Li LC and Dahiya R (2002). MethPrimer: designing primers for methylation PCRs. Bioinformatics 18: 1427-1431.

Nakayama M, Wada M, Harada T, Nagayama J, et al. (1998). Hypomethylation status of CpG sites at the promoter region and overexpression of the human MDR1 gene in acute myeloid leukemias. Blood 92: 4296-4307.

Reed K, Hembruff SL, Sprowl JA and Parissenti AM (2010). The temporal relationship between $A B C B 1$ promoter hypomethylation, $A B C B 1$ expression and acquisition of drug resistance. Pharmacogenomics J. 10: 489-504.

Suzuki MM and Bird A (2008). DNA methylation landscapes: provocative insights from epigenomics. Nat. Rev. Genet. 9: $465-476$.

Toyota M, Kopecky KJ, Toyota MO, Jair KW, et al. (2001). Methylation profiling in acute myeloid leukemia. Blood 97: 2823-2829.

Ueda K, Pastan I and Gottesman MM (1987). Isolation and sequence of the promoter region of the human multidrugresistance (P-glycoprotein) gene. J. Biol. Chem. 262: 17432-17436.

Wise JG (2012). Catalytic transitions in the human MDR1 P-glycoprotein drug binding sites. Biochemistry 51: 5125-5141.

Wittwer CT, Reed GH, Gundry CN, Vandersteen JG, et al. (2003). High-resolution genotyping by amplicon melting analysis using LCGreen. Clin. Chem. 49: 853-860.

Wojdacz TK and Dobrovic A (2007). Methylation-sensitive high resolution melting (MS-HRM): a new approach for sensitive and high-throughput assessment of methylation. Nucleic Acids Res. 35: e41.

Wojdacz TK, Borgbo T and Hansen LL (2009). Primer design versus PCR bias in methylation independent PCR amplifications. Epigenetics 4: 231-234. 